doi:10.32620/oikit.2018.81.14

$\begin{array}{ll}\text { УдК } 539.3 & \text { В. Ю. Мирошников }{ }^{1}, \text { Т. В. Денисова } \\ & \text {, В. С. Проценко }\end{array}$

\title{
Граничные условия контактного типа в задаче о круговой цилиндрической полости в упругом пространстве
}

\author{
${ }^{1}$ Харьковский национальный университет строительства и архитектуры \\ ${ }^{2}$ Харьковский национальный экономический университет им. Семена Кузнеца \\ ${ }^{3}$ Национальный аэрокосмический университет им. Н.Е. Жуковского \\ «Харьковский авиационный институт»
}

\begin{abstract}
Рассмотрена трехмерная задача теории упругости, когда на цилиндрической полости в упругом пространстве заданы условия контактного типа (нормальные перемещения и касательные напряжения). Решение получено на основе метода Фурье относительно уравнений Ламе в цилиндрических координатах. Доказана разрешимость и единственность решения задачи для данных граничных условий. Найдены нормальные и касательные напряжения в упругом теле. Проведено численное сравнение влияния граничных условий в виде перемещений и условий контактного типа на напряженное состояние упругого пространства.
\end{abstract}

Ключевые слова: цилиндрическая полость в пространстве, метод Фурье, граничные условия контактного типа.

\section{Введение}

Изученные ранее в работах [1 - 4] задачи о круговой цилиндрической полости в упругом пространстве относятся к основным задачам теории упругости (на границе полости заданы напряжения или перемещения). Граничные условия контактного типа, когда заданы нормальные перемещения и касательные напряжения, насколько известно авторам статьи, ранее никем не изучались. Между тем, можно привести примеры таких задач [5], возникающих при прочностных расчетах деталей машин, когда круглый жесткий вал контактирует без трения с упругим телом, будучи вставленным в полость с натягом. При проектировании таких узлов машин необходимо выяснить напряженно-деформированное состояние упругого тела (в идеализированном случае пространства с полостью) в условиях контактного взаимодействия. В частности, важно знать контактные напряжения, возникающие между контактирующими деталями, и на основе этих знаний обеспечить контактную прочность и жесткость.

\section{1. Постановка задачи}

Будем рассматривать задачу теории упругости для пространства с круговой цилиндрической полостью при краевых условиях:

$$
U_{\rho}(R, \varphi, z)=U_{0}(\varphi, z), \tau_{\rho \phi}(R, \varphi, z)=\tau_{1}(\varphi, z), \tau_{\rho z}(R, \varphi, z)=\tau_{2}(\varphi, z),
$$

где $\quad R$-радиус полости;

$U_{\rho}, \tau_{\rho \varphi}, \tau_{\rho z}-$ соответственно радиальное перемещение и касательные напряжения;

$$
U_{0}(\varphi, z), \tau_{1}(\varphi, z), \tau_{2}(\varphi, z) \text { - заданные, быстро убывающие к нулю по }
$$
переменной $z$, функции. 
Нужно найти решение уравнения Ламе [1] при краевых условиях (1) и определить напряжения в упругом пространстве.

Будем искать решение, которое дает напряжения, стремящиеся к нулю на бесконечности. В отношении заданных функций будем предполагать, что $U_{0}-$ непрерывна, $\tau_{k}$ - непрерывны или кусочно-непрерывны, они разлагаются в интегралы Фурье по переменной z и в ряды Фурье по координате $\varphi$.

Для них имеют место разложения

$$
U_{0}=\int_{-\infty}^{\infty} \sum_{m=-\infty}^{\infty} a_{m}(\lambda) e^{i(\lambda z+m \varphi)} d \lambda,\left(\begin{array}{c}
\tau_{1} \\
\tau_{2}
\end{array}\right)=\int_{-\infty}^{\infty} \sum_{m=-\infty}^{\infty}\left(\begin{array}{c}
b_{m}^{(1)}(\lambda) \\
b_{m}^{(2)}(\lambda)
\end{array}\right) e^{i(\lambda z+m \varphi)} d \lambda
$$

\section{2. Метод решения}

Искомый вектор перемещения $\stackrel{1}{U}$ представим в виде разложения

$$
\stackrel{\mathrm{r}}{U}(M)=\sum_{k=1}^{3} \int_{-\infty}^{\infty} \sum_{m=-\infty}^{\infty} B_{k, m}(\lambda) \stackrel{\mathrm{r}}{S_{k, m}}(M ; \lambda) d \lambda,
$$

где $M=M(\varphi, \rho, z)$ - точка в пространстве вне полости, а $\stackrel{1}{S}_{k, m}$ - базисные внешние решения взяты из книги [5] и обладают тем свойством, что стремятся к нулю когда $\rho \rightarrow \infty$.

Граничные условия (1) приводят к системе уравнений относительно неизвестных $B_{k, m}(\lambda)$ :

$$
\begin{aligned}
& B_{1, m} \cdot K_{m}^{\prime}(x)-B_{2, m} \cdot(\chi-D) K_{m}^{\prime}(x)-B_{3, m} \cdot m \cdot x^{-1} K_{m}(x)=a_{m}(\lambda) \\
& B_{1, m} \cdot 2 m D\left(x^{-1} K_{m}(x)\right)+B_{2, m} \cdot\left[\left(b_{m}+\gamma D\right) K_{m+1}(x)-\left(a_{m}+\gamma D\right) K_{m-1}(x)\right]+ \\
& +B_{3, m} \cdot\left[(1-D) K_{m}^{\prime}(x)-m^{2} x^{-1} K_{m}(x)\right]=b_{m}^{(1)}(\lambda) \cdot R \cdot G^{-1}(-i)(\operatorname{sign} \lambda)^{m+1} \\
& B_{1, m} \cdot 2 D \cdot K_{m}(x)+B_{2, m} \cdot 2\left(c_{m}+\delta D\right) K_{m}(x)-B_{3, m} \cdot m K_{m}(x)= \\
& =b_{m}^{(2)}(\lambda) R \cdot G^{-1}(-i)(\operatorname{sign} \lambda)^{m} . \\
& \quad \text { В равенствах (4) } K_{m}(x)-\text { функция Макдональда, } \gamma=2 \sigma-3, \delta=2(\sigma-1), \\
& \sigma \quad \text { коэфрициент Пуассона, } \chi=3-4 \sigma, \quad D=x \frac{d}{d x}, \quad x=|\lambda| R, \quad c_{m}=m^{2}+x^{2}, \\
& a_{m}=(m-1)(m-1+2 \sigma)+x^{2}, b_{m}=(m+1)(m+1-2 \sigma)+x^{2} .
\end{aligned}
$$

Определитель системы (4) обозначим через $D_{m}(\lambda)$.

При $m=0$ имеем

$$
D_{0}(\lambda)=8(1-\sigma) x^{2} K_{1}^{2}(x) K_{2}(x) .
$$

При $m \geq 1$ вычисления приводят к выражению 


$$
\begin{aligned}
& D_{m}(\lambda)=4 m^{2} K_{m}(x)\left[K_{m}^{2}(x)-K_{m-1}(x) K_{m+1}(x)\right]+4(1-\sigma) \times \\
& \times\left[m K_{m}(x)+x K_{m-1}(x)\right] \cdot\left[4 \cdot K_{m-1}(x) K_{m+1}(x)+x K_{m}(x)\left(K_{m-1}(x)+K_{m+1}(x)\right)\right],
\end{aligned}
$$
которое после несложных преобразований с использованием свойств функций $K_{m}(x)$ можно записать в виде

$$
D_{m}(\lambda)=4(1-2 \sigma)\left(x^{2}+m^{2}\right) K_{m-1}(x) K_{m}(x) K_{m+1}(x)+\varepsilon,
$$

где через $\varepsilon$ обозначены положительные величины.

Из (5) следует неравенство

$$
D_{m}(\lambda)>4(1-2 \sigma)\left(x^{2}+m^{2}\right) K_{m-1}(x) K_{m}(x) K_{m+1}(x) .
$$

Это неравенство справедливо и для $m \leq-1$.

Значит, система (4) имеет единственное решение при $\lambda \neq 0$ :

$$
\begin{aligned}
& B_{1, m}(\lambda)=a_{m}(\lambda) \cdot \Delta_{11}^{(m)}-k\left[b_{m}^{(1)}(\lambda) \cdot \Delta_{21}^{(m)} \cdot \operatorname{sign} \lambda+b_{m}^{(2)}(\lambda) \cdot \Delta_{31}^{(m)}\right] \cdot D_{m}^{-1}(\lambda), \\
& B_{2, m}(\lambda)=a_{m}(\lambda) \cdot \Delta_{12}^{(m)}-k\left[b_{m}^{(1)}(\lambda) \cdot \Delta_{22}^{(m)} \cdot \operatorname{sign} \lambda+b_{m}^{(2)}(\lambda) \cdot \Delta_{32}^{(m)}\right] \cdot D_{m}^{-1}(\lambda), \\
& B_{3, m}(\lambda)=a_{m}(\lambda) \cdot \Delta_{13}^{(m)}-k\left[b_{m}^{(1)}(\lambda) \cdot \Delta_{23}^{(m)} \cdot \operatorname{sign} \lambda+b_{m}^{(2)}(\lambda) \cdot \Delta_{33}^{(m)}\right] \cdot D_{m}^{-1}(\lambda),
\end{aligned}
$$

где $k=i \cdot R \cdot G^{-1}(\operatorname{sign} \lambda), \Delta_{k r}^{(m)}$ - алгебраические дополнения соответствующих элементов матрицы.

Остается подставить их в (3). На этом формальное решение задачи можно считать завершенным. Вектор $U$, определенный равенством (3), удовлетворяет всем условиям задачи.

Для обоснования формального решения достаточно установить для (3) справедливость предельных переходов при $\rho \rightarrow R$ в выражениях $U_{\rho}, \tau_{\rho \varphi}, \tau_{\rho z}$ и законность вычисления и непрерывность производных до второго порядка вклю-

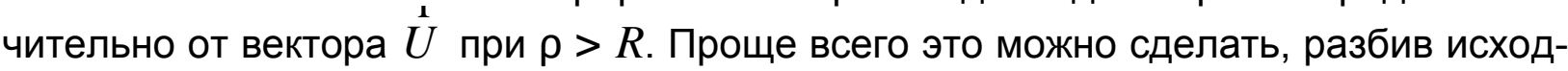
ную задачу на три вспомогательные: $U_{0} \neq 0, \tau_{1}=\tau_{2} \equiv 0 ; U_{0}=\tau_{2} \equiv 0, \tau_{1} \neq 0$; $U_{0}=\tau_{1} \equiv 0, \tau_{2} \neq 0$.

Дальше дело сводится к установлению равномерной сходимости рядовинтегралов, полученных из (3), для $U_{\rho}, \tau_{\rho \varphi}, \tau_{\rho z}$ в области $\rho \geq R$.

Анализ алгебраических дополнений $\Delta_{k r}^{(m)}$ для коэффициентов $B_{k, m}(\lambda)$ при $\lambda \rightarrow 0$ и $\lambda \rightarrow \infty$ вместе с неравенством (6) позволяет заключить, что все формальные действия, выполненные при нахождении решения (3), являются законными при условии сходимости рядов-интегралов:

$$
\sum_{m=-\infty}^{\infty} \int_{-\infty}^{\infty}\left|a_{m}(\lambda)\right|(1+x+|m|) d \lambda, \quad \sum_{m=-\infty}^{\infty} \int_{-\infty}^{\infty}\left|b_{m}^{(k)}(\lambda)\right|\left(1+x^{2}+m^{2}\right) d \lambda, k=1,2 .
$$


Наибольшую трудность при этом представляет установление фракта принадлежности вектора $\stackrel{L}{U}$ множеству $C^{2}(\Omega)$, где $\Omega=\{\rho, \rho>R\}$. На этом этапе доказательства используется прием, предложенный в [3]. Он основан на теореме сложения модифицированных функций Бесселя и неравенстве [3]:

$$
I_{m}(x) K_{m}(x)>\frac{C}{m^{2}(1+x)}, m \neq 0, x>0 .
$$

\section{3. Численные результаты и их сравнение}

Имеется цилиндрическая полость в упругом пространстве, на границах которой заданы условия контактного типа $U_{0}(\varphi, z)=10^{-4} \cdot\left(z^{2}+10^{2}\right)^{2}$, $\tau_{\rho \varphi}=\tau_{\rho z}=0$. Пространство - упругий материал, коэффициент Пуассона $\sigma=0,35$, модуль упругости $E=2 \kappa H / c^{2}$. Радиус цилиндрической полости $R=10 \mathrm{~cm}$.

Ввиду симметрии задача решалась при $m=0$, границы интегрирования были приняты $(-1,1)$. Вычисление интегралов выполнено по квадратурным фрормулам Филона. Граничные условия при заданных параметрах выполняются с точностью $10^{-5}$.

Для оценки влияния граничных условий на упругое пространство при тех же самых параметрах была рассчитана задача с заданными на границах полости перемещениями [6] $U_{\rho}(\varphi, z)=10^{-4} \cdot\left(z^{2}+10^{2}\right)^{2}, U_{\phi}(\varphi, z)=U_{z}(\varphi, z)=0$.

Графики нормальных напряжений $\sigma_{\rho}$ с заданными перемещениями и заданными условиями контактного типа изображены на рис. 1.

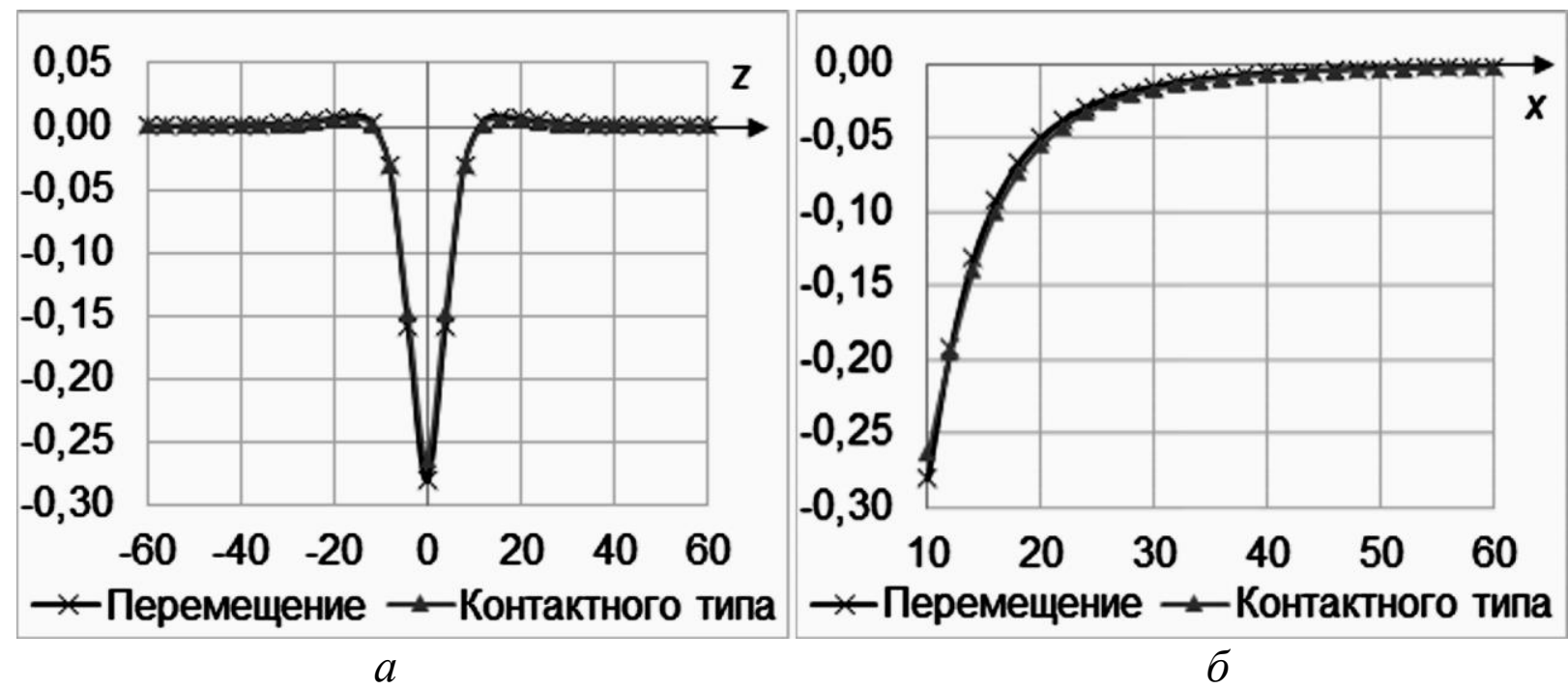

Рис. 1. Напряжения $\sigma_{\rho}: a-$ вдоль оси $z ; \sigma-$ вдоль оси $x$

На рис. 1, а показаны напряжения $\sigma_{\rho}$ вдоль оси $z$. Напряжения от воздействия граничных условий в виде перемещения больше, чем от граничных условий 
контактного типа. Так, максимальные напряжения от воздействия перемещений $\sigma_{\rho}^{(n)}=-0,28 \kappa \mathrm{H}_{\mathrm{cm}} \mathrm{cm}^{2}$ от воздействия условий контактного типа $\sigma_{\rho}^{(\kappa)}=-0,263 \kappa H / c \mu^{2}$. Кроме того, имеется эффрект для обоих рассматриваемых граничных условий: когда угол касательной от заданной функции перемещения к оси $x$ становится меньше $45^{\circ}$ (при $z=12$ см), напряжения становятся положительными (происходит растяжение).

На рис. 1, $\sigma$ показаны напряжения $\sigma_{\rho}$ вдоль оси $x$ начиная от поверхности цилиндрической полости. По мере удаления от цилиндрической полости происходит затухание напряжений. Несмотря на меньшие максимальные напряжения на поверхности цилиндра при граничных условиях контактного типа $\left(\sigma_{\rho}^{(\kappa)}=-0,263 \kappa H / c \mu^{2}\right.$ против $\left.\sigma_{\rho}^{(n)}=-0,28 \kappa H / c \mu^{2}\right)$, затухание напряжений в этом случае происходит медленнее. Так, на расстоянии $x=12$ см напряжения от условий контактного типа уже больше напряжений от граничных условий в виде перемещений: $\sigma_{\rho}^{(\kappa)}=-0,195 \kappa H / c \mu^{2}, \sigma_{\rho}^{(n)}=-0,192 \kappa H / c м^{2}$.

Графики нормальных напряжений $\sigma_{\varphi}$ с заданными перемещениями и заданными условиями контактного типа изображены на рис. 2.

На рис. 2, а показаны напряжения $\sigma_{\varphi}$ вдоль оси $z$. В области $-6<z<6$ напряжения $\sigma_{\varphi}$ от условий контактного типа меньше, чем от граничных условий с перемещениями, однако при $z>|6| \sigma_{\varphi}^{(n)}<\sigma_{\varphi}^{(\kappa)}$. Кроме того, имеется эффрект для обоих рассматриваемых граничных условий: в точке максимальных заданных перемещений $(z=0)$ напряжения $\sigma_{\varphi}$ снижаются. $К$ примеру, при $z=0$ $\sigma_{\varphi}^{(n)}=0,077 \kappa H / c M^{2}$, а при $z=4 \sigma_{\varphi}^{(n)}=0,085 \kappa H / c M^{2}$, далее при увеличении $z$ напряжения $\sigma_{\varphi}$ снижаются для обоих рассматриваемых граничных условий.

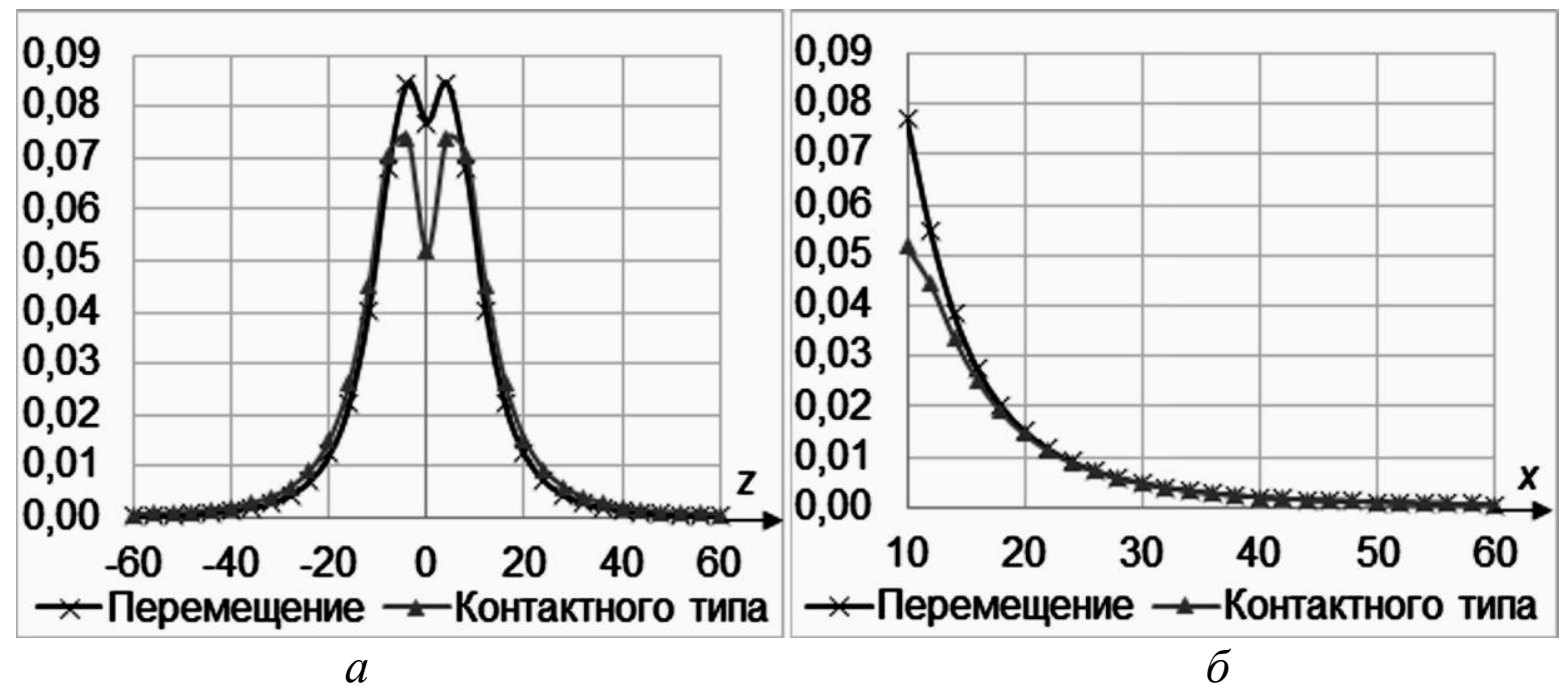

Рис. 2. Напряжения $\sigma_{\varphi}:$ - вдоль оси $z ; \sigma-$ вдоль оси $x$ 
На рис. 2, б показаны напряжения $\sigma_{\varphi}$ вдоль оси $x$ начиная от поверхности цилиндрической полости. По мере удаления от цилиндрической полости происходит затухание напряжений. При этом напряжения от условий контактного типа меньше, чем напряжения от граничных условий с перемещениями.

Графики нормальных напряжений $\sigma_{z}$ с заданными перемещениями и заданными условиями контактного типа представлены на рис. 3.

На рис. 3, а показаны напряжения $\sigma_{z}$ вдоль оси z. Отрицательные напряжения наблюдаются на интервале $-6<z<6$. При $z>|6|$ напряжения $\sigma_{z}$ становятся положительными. При этом напряжения от условий контактного типа существенно больше, чем напряжения от граничных условий с перемещениями.

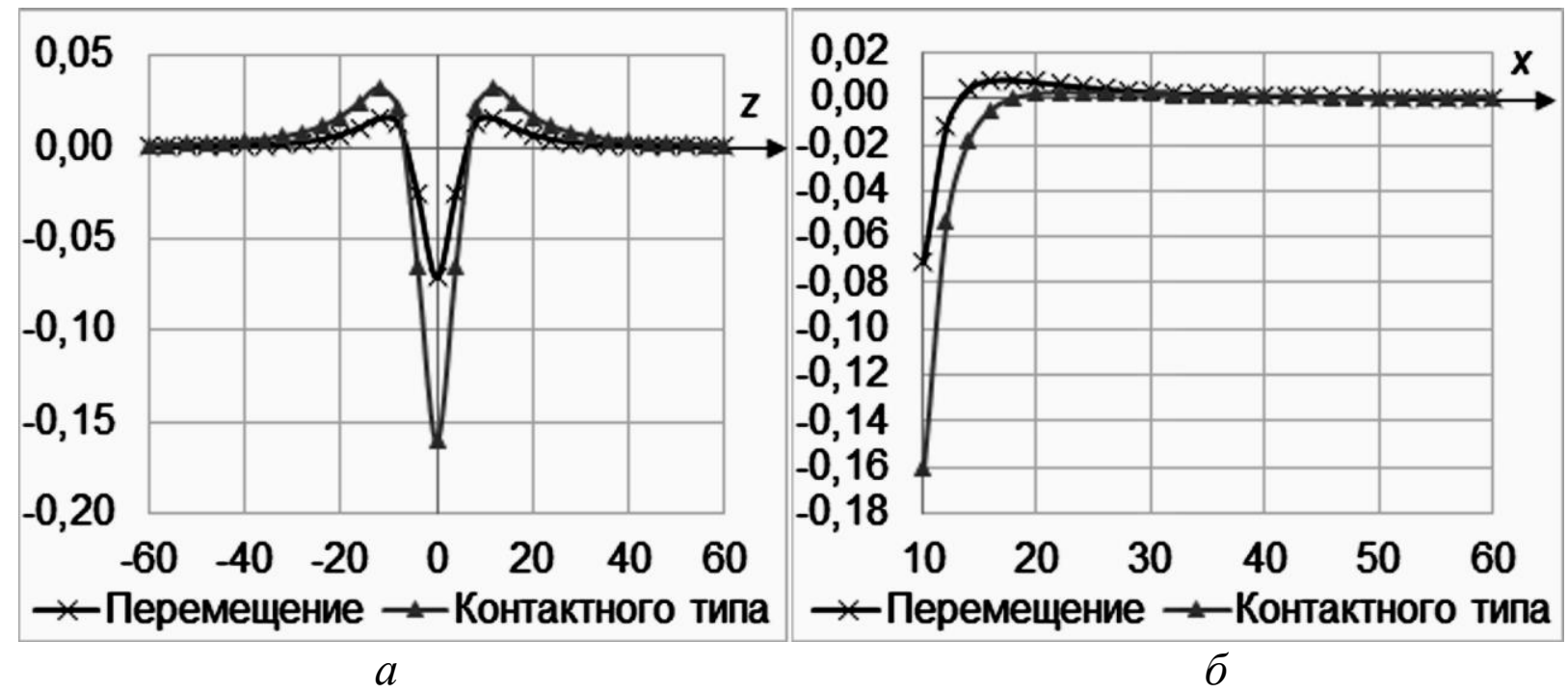

Рис. 3. Напряжения $\sigma_{z}:$ a - вдоль оси $z ; \sigma-$ вдоль оси $x$

На рис. 3, б показаны напряжения $\sigma_{z}$ вдоль оси $x$ начиная от поверхности цилиндрической полости. Для условий с перемещениями напряжения $\sigma_{z}$ становятся растягивающими при $x>13 \mathrm{~cm}$, для условий контактного типа при $x>18 \mathrm{~cm}$. Сжатая зона при условиях контактного типа существенно больше, при этом почти отсутствует растянутая зона.

Касательные напряжения $\tau_{\rho \varphi}$ и $\tau_{\varphi z}$ равны нулю, поэтому графики этих напряжений опущены.

Графики касательных напряжений $\tau_{\rho z}$ с заданными перемещениями и заданными условиями контактного типа изображены на рис. 4.

На рис. 4, а показаны напряжения $\tau_{\rho z}$ вдоль оси $z$. При заданных перемещениях они не равны нулю и имеют максимальное значение на расстоянии $z= \pm 5 \mathrm{~cm}: \tau_{\rho z}=$ m0,0397 $\kappa \mathrm{H} / \mathrm{cm}^{2}$. При заданных условиях контактного типа напряжение $\tau_{\rho z}$ задано и равно нулю. 


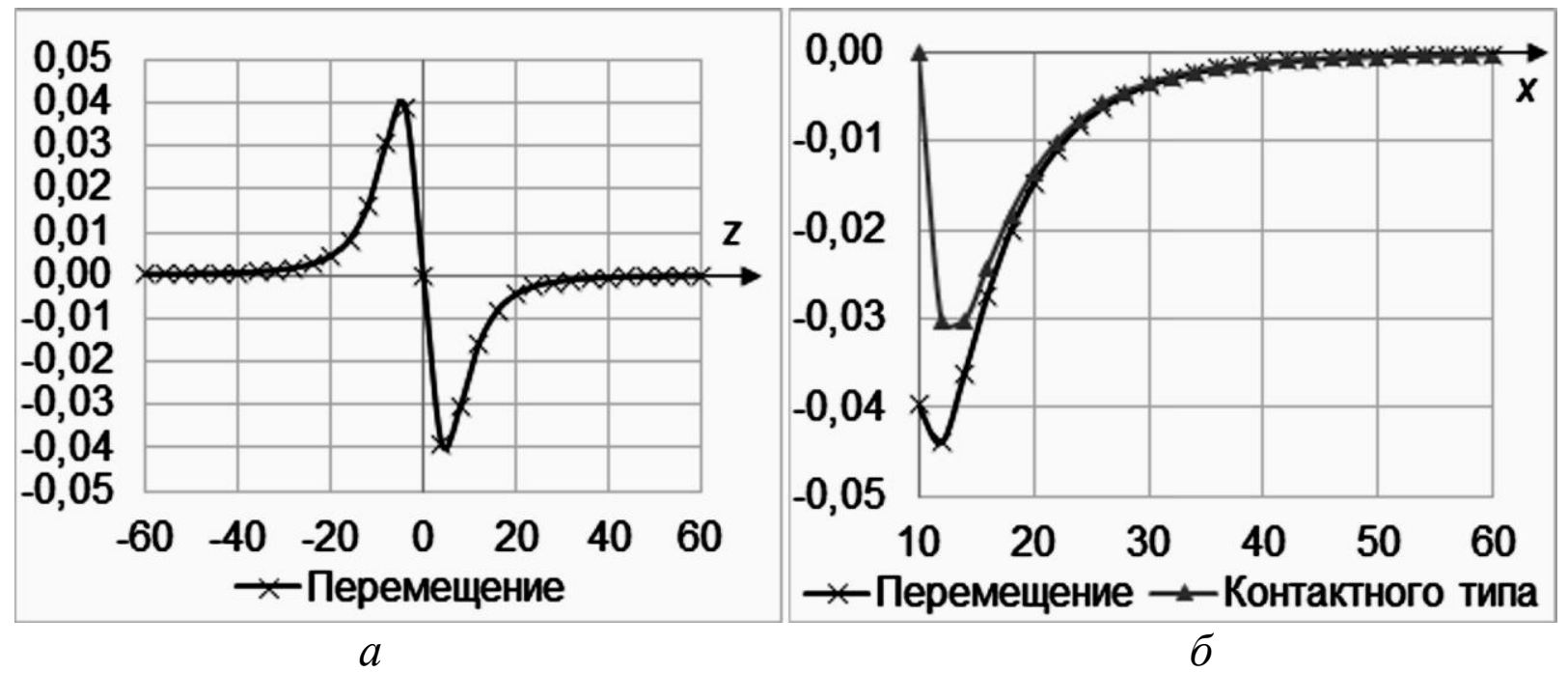

Рис. 4. Напряжения $\tau_{\rho z}$ : $а$ - вдоль оси $z ; \sigma-$ вдоль оси $x$, при $z=5$ см

На рис. 4, $б$ показаны напряжения $\tau_{\rho z}$ вдоль оси $x$ начиная от поверхности цилиндрической полости при $z=5$ см (при $\left.z=0 \tau_{\rho z}=0\right)$ ). Касательные напряжения $\tau_{\rho z}$ от воздействия граничных условий в виде перемещений больше, чем от граничных условий контактного типа. Сначала (возле цилиндра) напряжения растут и достигают максимальных значений: $\tau_{\rho z}^{(n)}=\mathrm{m} 0,0445 \kappa H / \mathrm{cm}^{2}$ при $x=11 \mathrm{~cm} \mathrm{и}$ $\tau_{\rho z}^{(\kappa)}=\mathrm{m} 0,0317 \kappa H / \mathrm{cm}^{2}$ при $x=13 \mathrm{~cm}$. Далее по мере удаления от цилиндра напряжения снижаются.

\section{Выводы}

На основе метода Фурье получено точное решение задачи теории упругости, когда на цилиндрической полости в упругом пространстве заданы условия контактного типа (нормальные перемещения и касательные напряжения).

Доказана разрешимость и единственность решения системы уравнений на основе базисных решений уравнения Ламе для данных граничных условий.

Исследовано численное влияние граничных условий в виде перемещений и граничных условий контактного типа на напряженное состояние упругого пространства.

Построены графики, которые иллюстрируют различие между двумя напряженными состояниями при двух разных типах граничных условий.

\section{Литература}

1. Подильчук, Ю. Н. Трехмерные задачи теории упругости / Ю. Н. Подильчук.-Київ : Наук. Думка, 1979. - 240 с.

2. Соляник-Краса, К. В. Осесимметричная задача теории упругости / К. В. Соляник-Краса. - М. : Стройиздат, 1987. - 336 с.

3. Николаев, А. Г. Классы разрешимости методом Фурье основных краевых задач теории упругости для некоторых пространственных канонических областей / 
А. Г. Николаев. - Харьков : ХАИ, 1996. - 16 с. - Деп. в ГНТБ Украины 23.10.1996, № 1976 . - Ук 96.

4. Николаев, А. Г. Обобщенный метод Фурье в пространственных задачах теории упругости / А. Г. Николаев, В. С. Проценко. - Харьков : ХАИ, 2011. - 344 с.

5. Александров, В. М. Контактные задачи В машиностроении / В. М. Александров, Б. Л. Ромалис. - М. : Машиностроение, 1986. - 176 с.

6. Мірошніков, В. Ю. Друга основна задача теорії пружності в просторі 3 декількома паралельними круговими циліндричними порожнинами / В.Ю. Мірошніков // Збірник наукових праць Харківського національного університету Повітряних Сил. - 2017. - № 1 (50). - С. 92 - 96.

Поступила в редакцию 20.09.2018

Розглянуто тривимірну задачу теорії пружності, коли на циліндричній порожнині в пружному просторі задано умови контактного типу (нормальні переміщення й дотичні напруження). Розв'язок отримано на основі методу Фур'є щодо рівнянь Ламі в циліндричних координатах. Доведено однозначність розв'язання задачі для даних граничних умов. Знайдено нормальні і дотичні напруження в пружному тілі. Проведено числове порівняння впливу граничних умов у вигляді переміщень і граничних умов контактного типу на напружений стан пружного простору.

Ключові слова: циліндрична порожнина в просторі, метод Фур'є, граничні умови контактного типу.

A three-dimensional problem of the theory of elasticity is considered, when contact-type conditions (normal displacements and tangential stresses) are given on a cylindrical cavity in elastic space. The solution is obtained on the basis of the Fourier method with respect to the Lame equations in cylindrical coordinates. The solvability and uniqueness of the problem for these boundary conditions is proved. Normal and tangential stresses are found in the elastic body. A numerical comparison is made of the influence of the boundary conditions in the form of displacements and boundary conditions of the contact type on the stressed state of the elastic space.

Keywords: cylindrical cavity in space, Fourier method, boundary conditions of contact type. 